\title{
Knockdown of ARK5 expression suppresses invasion of ovarian cancer cells
}

\author{
SHUXIAO WANG ${ }^{1 *}$, SHUWEI LI $^{2 *}$, HUI WANG $^{1,3}$, WEI LI $^{4}$, YUXUE GAO $^{5}$, XUEJIAN WANG ${ }^{1}$, CHUNYAN FANG $^{1}$, \\ BAOGANG ZHANG ${ }^{5}$, XIUNING SUN $^{6}$, RUIFANG LI $^{6}$, WEIWEI SHI ${ }^{7}$, MEILING CHEN $^{1}$ and LIHONG SHI ${ }^{1}$ \\ Departments of ${ }^{1}$ Pharmacology and ${ }^{2}$ Physics, Weifang Medical University, Weifang, Shandong 261053; \\ ${ }^{3}$ Department of Obstetrics and Gynecology, Zhucheng People's Hospital, Zhucheng, Shandong 252300; \\ ${ }^{4}$ Department of Gynecology, Zhenjiang Maternity and Child Health Hospital, Zhenjiang, Jiangsu 212001; \\ Departments of ${ }^{5}$ Pathology, ${ }^{6}$ Microbiology and ${ }^{7}$ Chemistry, Weifang Medical University, Weifang, Shandong 261053, P.R. China
}

Received March 4, 2018; Accepted December 6, 2018

DOI: $10.3892 / \mathrm{mmr} .2019 .9901$

\begin{abstract}
The aim of the current study was to investigate the effects and the molecular mechanisms of ARK5 in ovarian cancer cell invasion. The plasmid pGCsilencerU6/GFP/Neo-RNAi-ARK5 and the control vector with a scramble sequence were transfected into SKOV3 cells to establish ARK5-deficient SKOV3 cells (siARK5/SKOV3) and a control cell line (Scr/SKOV3), respectively. Reverse transcription-polymerase chain reaction (RT-PCR) and Western blot analysis were used to determine the mRNA and protein expression levels of ARK5. Migration and invasion abilities of SKOV3 cells were determined in chemotaxis and invasion assays, respectively. The epidermal grow th factor-1 (EGF-1)-induced expression of matrix metallopeptidase (MMP)-2 and MMP-9, epithelial-mesenchymal transition (EMT) and phosphorylation of mechanistic target of rapamycin kinase (mTOR) in siARK5/SKOV3 and Scr/SKOV3 cells were detected by western blot. RT-PCR and western blot analyses demonstrated that the expression of ARK5 was significantly downregulated in siARK5/SKOV3 cells at the mRNA and protein levels $(\mathrm{P}<0.01)$. The migration and invasion abilities of siARK5/SKOV3 cells were markedly decreased compared with $\mathrm{Scr} / \mathrm{SKOV} 3$ cells $(\mathrm{P}<0.01)$. In addition, the results demonstrated that EGF-1-induced expression of MMP-2 and MMP-9, EMT and phosphorylation of mTOR were suppressed in siARK5/SKOV3 cells as compared with $\mathrm{Scr} / \mathrm{SKOV} 3$ cells $(\mathrm{P}<0.01)$. The
\end{abstract}

Correspondence to: Professor Lihong Shi, Department of Pharmacology, Weifang Medical University, 7,166 Baotongxi Street, Weifang, Shandong 261053, P.R. China

E-mail: 963587089@qq.com

*Contributed equally

Key words: AMPK-related protein kinase-5, ovarian cancer, SKOV3 cells, invasion, epithelial-mesenchymal transition current study demonstrated that ARK5 is a critical factor involved in SKOV3 cell invasion and ARK5 increases invasive potential by promoting EMT and activating the Akt-mTOR-MMPs pathway.

\section{Introduction}

Ovarian cancer is the leading cause of mortality among malignant gynecological tumors worldwide (1). In the US alone, $>20,000$ women are diagnosed ovarian cancer and there are $>14,000$ ovarian cancer-associated mortalities each year (2). The high mortality rate of ovarian cancer is attributed to its typically advanced stage at detection, and high invasive and metastatic malignancy. Despite efforts to develop approaches for ovarian cancer treatment, no treatment option has been proven to reduce the mortality of ovarian cancers (3). Therefore, it is urgent to develop novel therapeutic treatments for better control of ovarian cancers, and thus, understanding of the mechanisms responsible for ovarian cancer invasion and metastasis may provide potential therapeutic targets.

Recently, AMPK-related protein kinase-5 (ARK5), a crucial member of the human AMP-activated protein kinases (AMPKs) family, has been identified to be associated with metastasis of cancer cells (4). Metastasis is a complex multistep process involving cell migration and invasion. ARK5 has been reported to be an important participant in mediating cancer cell migration activity and its activation is caused by protein kinase B (Akt)-dependent phosphorylation (5). In addition, it has been demonstrated that high expression of ARK5 can accelerate tumor invasion and metastasis (6). However, mechanisms of ARK5 involved in ovarian cancer invasion and metastasis has not been fully ascertained.

The present study aimed to clarify the involvement of ARK5 in ovarian cancer invasion. The expression of ARK5 in ovarian cancer cell lines and tissues, and the biological impact of ARK5 on the invasive capabilities of ovarian cancer cells were investigated. In addition, mechanistic targets of ARK5 in modulating ovarian cancer invasion were explored in the current study. 


\section{Patients and methods}

Patients and tissue specimens. This study was conducted using 20 pairs of selected frozen (liquid nitrogen) ovarian cancer tissues $(\mathrm{T})$ and adjacent non-tumor ovarian tissues $(\mathrm{N})$. Details of clinical pathological features of the patients with ovarian cancer are presented in Table SI. Neither radiotherapy nor preoperative chemotherapy was received prior to tissue collection. All primary ovarian cancer tissues and corresponding adjacent nontumor ovarian tissues were identified by routine pathological observation. Institutional research ethics committee approval of Weifang Medical University (Shandong, China) and patient consent were obtained in advance of the use of these clinical specimens for research purposes.

Cell culture and reagents. Human ovarian cancer cell lines SKOV3 and OVCAR-3 were obtained from the American Type Culture Collection (Manassas, VA, USA). Ovarian cancer cell line. A2780 and normal ovarian epithelial cell line IOSE80 were obtained from Shanghai Huiying Biotechnology Co., Ltd. (Shanghai, China). All cells were cultured in RPMI-1640 (Hyclone, GE Healthcare Life Sciences, Logan, UT, USA) supplemented with $10 \%$ fetal calf serum from Zhejiang Tianhang Biotechnology Co., Ltd. (Zhejiang, China) and were maintained at $37^{\circ} \mathrm{C}$ in $5 \% \mathrm{CO}_{2}$ incubator. Membranes and chemotaxis chambers were from Neuro Probe, Inc. (Gaithersburg, MD, USA). The human epidermal growth factor-1 (EGF-1) was from R\&D Systems, Inc. (Minneapolis, MN, USA).

Plasmid construction, small interfering RNA (siRNA) and plasmid transfection. The cells $\left(2 \times 10^{5}\right.$ cells) were cultured in a $35 \mathrm{~mm}$ dish with serum-free medium for $24 \mathrm{~h}$ and then moved into complete medium for transfection. Two expression plasmids (200 ng/ $\mu \mathrm{l})$ containing a scrambled sequence (5'-CTCAACTTG ATCCTGTGAG-3') (pGCsilencerU6/GFP/Neo-RNAi-Scr) and a target sequence (5'-GAAGTTATGCTTTATTCAC-3'; pGCsilencerU6/GFP/Neo-RNAi-ARK5) were obtained from Shanghai GeneChem Co., Ltd. (Shanghai, China). The transfection was performed using Lipofectamine ${ }^{\circledR} 2000$ (Invitrogen; Thermo Fisher Scientific, Inc., Waltham, MA, USA). Stable transfection was selected using $500 \mu \mathrm{g} / \mathrm{ml}$ neomycin for at least 6 months (7).

Reverse transcription polymerase chain reaction (RT-PCR). ARK5 mRNA expression was determined by RT-PCR. The total RNA from SKOV3 cells was extracted using TRIzol (Thermo Fisher Scientific, Inc.). One Step RNA PCR kit (AMV) from Takara Biotechnology Co., Ltd. (Dalian, China) was used to reverse transcribe RNA to cDNA at $50^{\circ} \mathrm{C}$ for $30 \mathrm{~min}$ and $94^{\circ} \mathrm{C}$ for $2 \mathrm{~min}$. The $\beta$-actin primers (forward, 5'-ATGTTTGAGACC TTCAACAC-3' and reverse, 5'-CACGTCACACTTCATGAT GG-3') and ARK5 (forward, 5'-GACATGGTTCACATCAGA CGA-3' and reverse, 5'-CAATAGTGCACAGCAGAGACG-3') were synthesized using a Takara PrimeScript RT kit (Takara Biotechnology Co., Ltd) with reaction at $50^{\circ} \mathrm{C}$ for $30 \mathrm{~min}$, pre-denaturation at $94^{\circ} \mathrm{C}$ for $2 \mathrm{~min}, 30$ cycles of denaturation at $94^{\circ} \mathrm{C}$ for $30 \mathrm{sec}$, annealing at $60^{\circ} \mathrm{C}$ for $30 \mathrm{sec}$ and extension at $72^{\circ} \mathrm{C}$ for $1 \mathrm{~min}$ in order to produce amplification products which were then electrophoresed on $1.5 \%$ the agarose gel.
Western blotting. For western blot analysis, the whole-cell extracts were prepared in radioimmunoprecipitation assay buffer $(40 \mathrm{mM} \mathrm{NaF}, 20 \mathrm{mM}$ Tris, $2.5 \mathrm{mM}$ EDTA, $1 \%$ deoxycholate, $1 \%$ Triton X-100, 0.1\% SDS, $10 \mathrm{mM}$ $\mathrm{Na}_{4} \mathrm{P}_{2} \mathrm{O}_{7}$ and $1 \mathrm{mM}$ phenylmethylsulfonyl fluoride). Protein concentration was determined with BCA and $25 \mu 1$ per well of protein samples were separated by $10 \%$ SDS-PAGE, then transferred onto polyvinylidene difluoride membranes, immunoblotted with appropriate primary antibodies at $4^{\circ} \mathrm{C}$ overnight and secondary antibodies at room temperature for $1 \mathrm{~h}$. (goat anti-mouse IgG, cat. no. SA00001-1; 1:2,000 and goat anti-rabbit IgG, cat. no. SA00001-2, 1:2,000; Shanghai Biyuntian Biotechnology Co., Ltd), The chemiluminescent signals were detected using ECL Plus (WBKLS0100; EMD Millipore, Billerica, MA, USA) and finally visualized by Image Quant LAS 500 (GE Healthcare). Western blot analysis is representative of at least three independent repeated experiments. Densitometric analysis was used to quantify the protein bands and $\beta$-actin was used as normal control, with the protein bands being quantified using ImageJ software version 1.4.3.67 (National Institutes of Health, Bethesda, MD, USA). The following primary antibodies were used in this study: mTOR (cat. no. 2983; 1:1,000 dilution), phospho (p)-mTOR (cat. no. 2971; 1:1,000 dilution), ARK5 (ab71814; 1:500; Abcam, Cambridge, MA, USA), MMP-2 (cat. no. 4022; 1:1,000 dilution), MMP-9 (cat. no. 3852; 1:1,000 dilution), E-cadherin (cat. no. 3195; 1:1,000 dilution), N-cadherin (cat. no. 4061S; 1:1,000 dilution; all Cell Signaling Technology, Inc., Danvers, MA, USA) and $\beta$-actin (cat. no. sc-47778; 1:1,000 dilution; Santa Cruz Biotechnology, Inc., Dallas, TX, USA).

Chemotaxis assay. A chemotaxis assay was performed as described previously (8). Briefly, the chemotaxis ability of cells was measured using Transwell inserts with $8.0 \mathrm{~mm}$ pore polycarbonate membrane. The chemoattractant EGF-1 $(10 \mathrm{ng} / \mathrm{ml})$ was loaded into the lower chemotaxis chamber with serum free RPMI 1640 medium and $5 \times 10^{5}$ cells $/ \mathrm{ml}$ cells suspended in the binding medium [RPMI-1640, $0.1 \%$ bovine serum albumin (BSA) and $25 \mathrm{mM}$ HEPES] were added into the upper chambers. After $3 \mathrm{~h}$, the non-migrating cells were removed by wiping the upper side of the membrane, and the migrating cells were fixed in $4 \%$ paraformaldehyde for $10 \mathrm{~min}$ and then stained with $0.1 \%$ crystal violet at room temperature for $15 \mathrm{~min}$. The number of cells on the lower surface, which had migrated through the membrane, was counted under a light microscope in at least five random fields at a magnification of $x 400$. Chemotaxis index (CI) was defined by the ratio of the number of cells on the lower surface of the membrane in the experimental group to the number of corresponding cells in the control group. All assays were repeated at least three times independently.

Matrigel invasion assay. The invasion of SKOV3 ovarian cells in vitro was evaluated using Matrigel-coated Transwell inserts (Corning Incorporated, Corning, NY, USA) as described previously (9). Briefly, the Transwell inserts with $8 \mathrm{~mm}$ pore size were coated with a final concentration of $1.5 \mathrm{mg} / \mathrm{ml}$ Matrigel. The Matrigel contains collagen type IV, heparin sulfate proteoglycan, entactin and laminin. Cell suspension $\left(5 \times 10^{5} \mathrm{cells} / \mathrm{ml}\right.$ in $\left.200 \mu \mathrm{l}\right)$ were plated on the surface of top 
chamber and $300 \mu \mathrm{l}$ binding medium (RPMI-1640, 0.1\% BSA and $25 \mathrm{mM}$ HEPES) with $10 \mathrm{ng} / \mathrm{ml}$ of EGF-1 was added into the lower well. The assembly was incubated for $24 \mathrm{~h}$ at $37^{\circ} \mathrm{C}$ in humidified $5 \% \mathrm{CO}_{2}$. The invasive cells on the lower surface of the membrane were fixed in $4 \%$ paraformaldehyde for 10 min and stained with $0.1 \%$ crystal violet at room temperature for $30 \mathrm{~min}$. The number of invading cells was counted under a light microscope in five random fields at a magnification of $\mathrm{x} 400$. All assays were repeated at least three times independently.

Statistical analysis. The results are presented as the mean \pm standard deviation. Each experiment was performed at least three times. The statistical difference of data between multiple groups was analyzed by one-way analysis of variance. The Fisher's LSD test was used to determine significance among the multiple groups. All statistical analyses were carried out using the SPSS 17.0 statistical software (SPSS, Inc., Chicago, IL, USA). $\mathrm{P}<0.05$ was considered to indicate a statistically significant difference.

\section{Results}

Increased expression of ARK5 in ovarian cancer cell lines and tissues. To reveal the role of ARK5 in human ovarian cancer, western blot analysis was performed to evaluate ARK5 expression in human ovarian cancer cell lines and primary cancer tissues. The results demonstrated that the protein levels of ARK5 in ovarian cancer cell lines, OVCAR-3, A2780 and SKOV3 were significantly increased compared with the normal ovarian epithelial cells line, IOSE80. SKOV3 cells exhibited the highest ARK5 protein expression, thus, SKOV3 cells were selected for used in further experiments (Fig. 1A and B). Meanwhile, ARK5 was also strongly up regulated in ovarian cancer tissues compared with paired adjacent non-cancerous tissues (Fig. 1C and D). Taken together, the results demonstrated that the expression of ARK5 was markedly upregulated in human ovarian cancer cells and clinical primary human ovarian cancer tissues.

Knockdown of ARK5 in ovarian cancer SKOV3 cells. To confirm the role of ARK5 in ovarian cancer cell metastasis, an RNA interference (RNAi) expression vector was designed to target human ARK5 in SKOV3 cells, with a scrambled sequence vector used as a control. RNAi-mediated ARK5 knockdown SKOV3 (siARK5/SKOV3\#1 and siARK5/SKOV3\#2) and the RNAi control SKOV3 (Scr/SKOV3) cell lines were produced. PCR and western analysis tests demonstrated that ARK5 was markedly reduced in siARK5/SKOV3\#1 and siARK5/SKOV3\#2 cells. siARK5/SKOV3\#2 was named 'siARK5/SKOV3' cells and used in the subsequent assays (Fig. 1E-H).

ARK5 knockdown impairs migration of ovarian cancer cells. Cell migration is essential for cancer metastasis (10). A chemotaxis assay was performed to identify whether ARK5 influences SKOV3 cells migration. The results revealed similar chemotaxis in parental SKOV3 and Scr/SKOV3 cells, and EGF-1 induced the robust chemotaxis of parental SKOV3 and Scr/SKOV3 cells with typical bell-shaped response curves with increasing doses. Chemotaxis was decreased in siARK5/SKOV3 cells compared with Scr/SKOV3 cells (Fig. 2A). The chemotaxis results indicated that ARK5 has a role in the chemotaxis of SKOV3 cells and downregulation of ARK5 impaired cell migration. The materials and methods states that the change in cell proliferation, however, did not interfere with the chemotaxis of SKOV3 cells in the present study, because it took $<3 \mathrm{~h}$ to finish the chemotaxis assay, which is far shorter than the cell doubling time.

ARK5 knockdown impairs SKOV3 cell invasion. Cell migration is crucial in tumor invasion, this whether ARK5 can influence ovarian cancer cell invasion was investigated in the current study $(11,12)$. EGF-1 $(10 \mathrm{ng} / \mathrm{ml})$ was used as a chemoattractant to stimulate the cells to penetrate through Matrigel and filters. Compared with the Scr/SKOV3 cells, the number of invading siARK5/SKOV3 cells was dramatically decreased. This result indicates that ARK5 has a role in SKOV3 cell invasion, and ARK5 knockdown results in reduced invasive ability (Fig. 2B and C).

ARK5 knockdown reduces the expression of $M M P-2$ and MMP-9 in SKOV3 cells. It is established that MMPs are involved in tumor invasion (13), therefore, MMP-2 and MMP-9 expression was assessed in siARK5/SKOV3 and control cells (14). Western blot analysis demonstrated that MMP-2 and MMP-9 expression was lower in siARK5/SKOV3 cells than in Scr/SKOV3 cells. In addition, EGF-1 (10 ng/ml) stimulation induced increased expression of MMP-2 and MMP-9 in Scr/SKOV3 cells; however, there was no obvious increase of MMP-2 and MMP-9 in siARK5/SKOV3 cells (Fig. 3A and B). These results suggest that downregulation of ARK5 was associated with reduced expression of MMP-2 and MMP-9 in ovarian cancer SKOV3 cells, indicating that MMP-2 and MMP-9, at least partially, have important roles in the invasiveness of ovarian cancer cells induced by ARK5.

Knockdown of ARK5 inhibited cell mesenchymal properties and reversed EMT. EMT is a vital process for metastasis of cancers, during which epithelial tumor cells acquire a more motile and invasive phenotype (15). In the current study, the expression of EMT markers was analyzed to address the mechanism of ARK5-facilitated ovarian cancer invasion. As shown in, the expression of mesenchymal marker $\mathrm{N}$-cadherin was much higher in $\mathrm{Scr} / \mathrm{SKOV} 3$ cells than that in SiARK5/SKOV3 cells whereas the expression of epithelial marker E-cadherin was much higher in SiARK5/SKOV3 cells than that in Scr/SKOV3 cells (Fig. 3C and D). Additionally, there were changes in cell morphology that are associated with decreased mesenchymal properties (Fig. 3E). siARK5/SKOV3 cells changed their morphology from an elongated, fibroblastic-like appearance to a cobblestone-like epithelial shape. Furthermore, EGF-1 (10 ng/ml) can increased the expression of $\mathrm{N}$-cadherin in Scr/SKOV3 cells, while there was only a marginal increase in $\mathrm{N}$-cadherin expression in siARK5/SKOV3 cells. These findings indicate that siARK5 reduces the expression of $\mathrm{N}$-cadherin and increases the expression of E-cadherin, which indicates that ARK5 may 

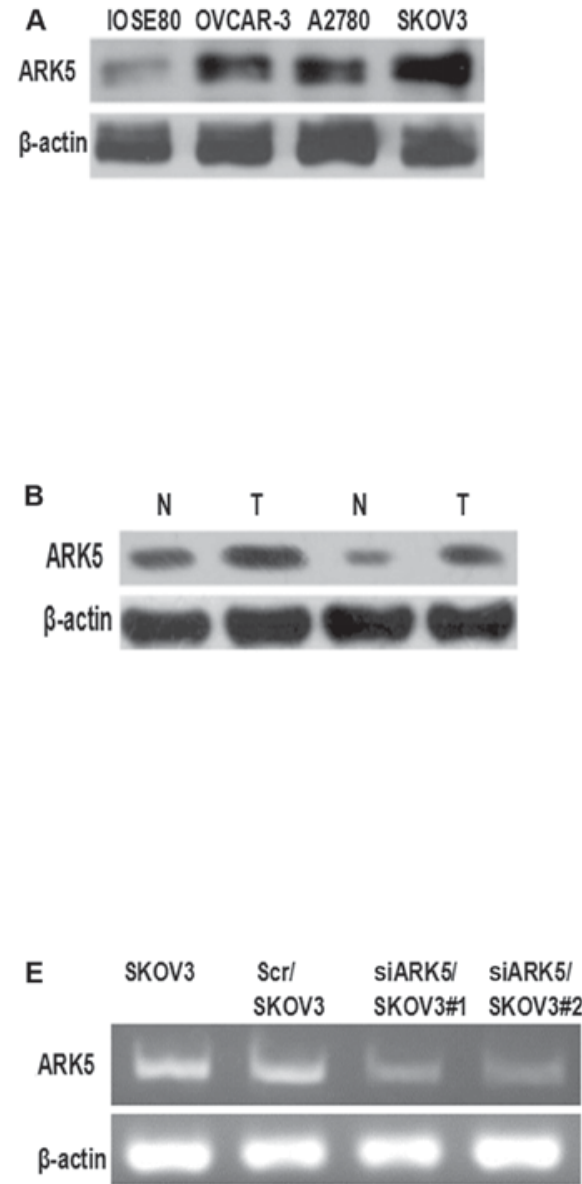

\section{G}

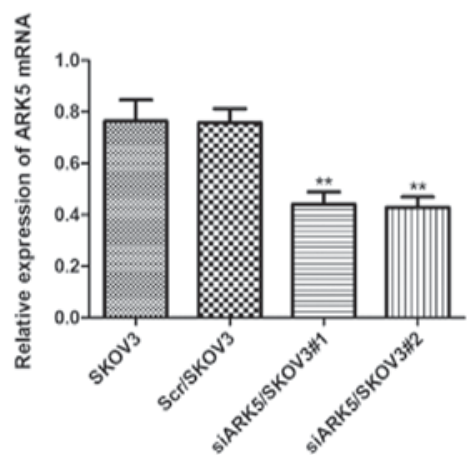

C

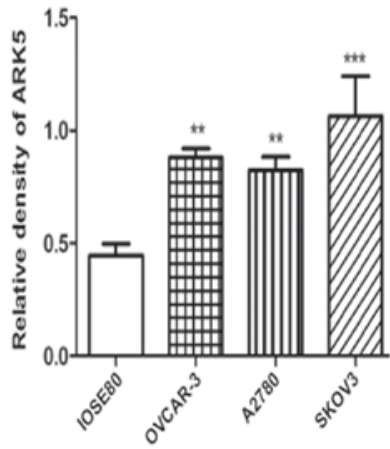

D

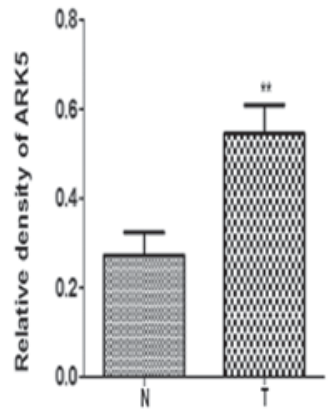

H

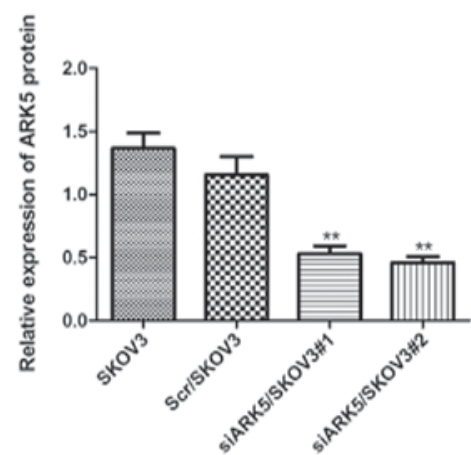

Figure 1. Increased ARK5 expression in ovarian cancer cell lines and ovarian cancer tissues, and ARK5 knockdown in SKOV3 cells. (A) Western blot analysis of ARK5 in ovarian cancer cell lines, OVCAR-3, A2780 and SKOV3, and normal ovarian epithelial cells line, IOSE80. (B) Western blot analysis of ARK5 in resected ovarian cancer tissues and adjacent normal ovarian tissues. (C) Densitometry analysis of ARK5 in ovarian cancer cell lines, OVCAR-3, A2780 and SKOV3, and normal ovarian epithelial cells line, ISOE80, ${ }^{* *} \mathrm{P}<0.01,{ }^{* * *} \mathrm{P}<0.001$ vs. IOSE80. (D) Densitometry analysis of ARK5 expression levels in resected ovarian cancer tissues and adjacent normal ovarian tissues. ${ }^{* *} \mathrm{P}<0.01$ vs. N. (E) Reverse transcription-polymerase chain reaction analysis of ARK5 mRNA expression in Scr/SKOV3 and siARK5/SKOV3 cells. (F) Western blot analysis of ARK5 protein expression in Scr/SKOV3 and siARK5/SKOV3 cells. (G) Statistical analysis of ARK5 mRNA expression in Scr/SKOV3 and siARK5/SKOV3 cells. ${ }^{* *} \mathrm{P}<0.01$ vs. SKOV3. (H) Densitometry analysis of ARK5 protein expression in Scr/SKOV3 and siARK5/SKOV3 cells. ${ }^{* *} \mathrm{P}<0.01$ vs. SKOV3. ARK5, AMPK-related protein kinase-5; N, adjacent normal tissue; T, ovarian cancer tissue; Scr, scramble control; siARK, ARK small interfering RNA.

promote EMT of ovarian cancer cell and knockdown ARK5 may reverse the EMT process (16).

ARK5 knockdown stabilized Akt-mTOR signaling pathway. Furthermore, it is well established that Akt activation can increase tumor invasion and metastasis in ovarian cancer (17). Multiple extracellular stimuli can induce the phosphorylation of Akt and mTOR downstream. The activation of the Akt-mTOR signaling pathway can promote the expression of
MMPs and the EMT process (18). However, the function of ARK5 in ovarian cancer invasion is not fully understood. To determine whether ARK5 is an upstream regulator of mTOR during ovarian cancer cell invasion, the effect of ARK5 on EGF-1-induced activation of mTOR was investigated in SKOV3 cells. In Scr/SKOV3 cells, phosphorylated mTOR was higher than in siARK5/SKOV3 cells (Fig. 4A and B). Notably, EGF-1 induced increased expression of p-mTOR in Scr/SKOV3 cells and siARK5/SKOV3 cells, whereas there was markedly lower 


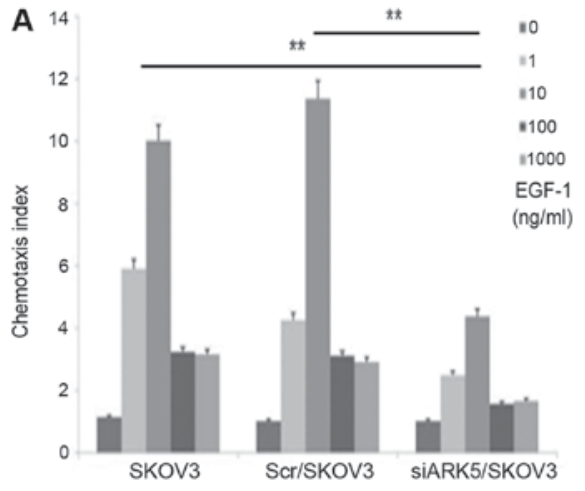

B
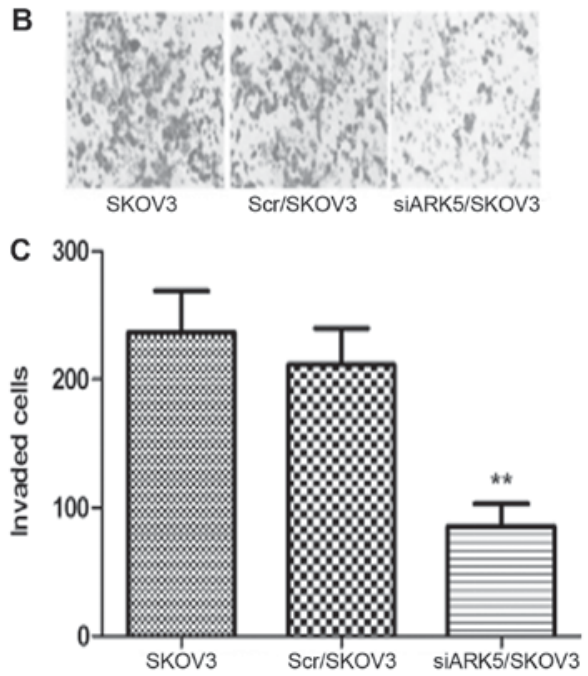

Figure 2. Reduction of ARK5 impairs SKOV3 cell invasion. (A) Chemotaxis index of SKOV3, Scr/SKOV3, siARK5/SKOV3 cells under different concentrations of EGF-1. ${ }^{* *} \mathrm{P}<0.01$. (B) Invasive ability of SKOV3, Scr/SKOV3, siARK5/SKOV3 cells. (C) Statistical analysis of invasive ability of SKOV3, Scr/SKOV3 and siARK5/SKOV3 cells. ${ }^{* *} \mathrm{P}<0.01$. EGF-1, epidermal growth factor-1; ARK5, AMPK-related protein kinase-5; Scr, scramble control siARK, ARK small interfering RNA.

increase in p-mTOR in siARK5/SKOV3 cells compared with $\mathrm{Scr} / \mathrm{SKOV} 3$ cells. These results indicated that EGF-1-induced mTOR phosphorylation was inhibited in siARK5/SKOV3 cells and ARK5 may be a key mediator of ovarian cell invasion via activation of the mTOR signaling pathway.

\section{Discussion}

ARK5 is a novel member of the AMPK family that has been reported to be crucial in mediating malignant activity of various cancers (19). ARK5 is directly activated by Akt and has critical roles in tumorigenesis, cancer invasion and metastasis $(20,21)$. Similar results have also been reported in ovarian cancer, with ARK5 upregulated in ovarian cancer tissues and is associated with poor prognosis $(22,23)$. However, the mechanism of ARK5 in facilitating ovarian cancer invasion has not been fully elucidated.

Understanding tumor invasion is an important issue for novel therapeutic applications and, thus, improving the clinical outcome of patients with ovarian cancer. In the current study, it was initially identified that ARK5 was highly expressed in ovarian cancer cell lines and primary ovarian cancer tissues, which corroborates a recent report by Phippen et al (23).
Polarized cell migration is a well-regulated process that is closely associated with the infiltration and invasion of tumors. Knockdown of ARK5 markedly impaired the chemotaxis and invasion ability of SKOV3 ovarian cancer cells. This result suggests that ARK5 may be a vital factor involved in the migration and invasion of ovarian cancer cells.

MMPs regulate invasion by degrading the extracellular matrix and basement membrane to promote invasion in various cancer types. In human pancreatic cancer, ARK5 has been reported to activate MMP-2 and MMP-9 via rapamycin-sensitive signaling (24). The findings of the current study demonstrated that ARK5 knockdown leads to repression of MMP-2 and MMP-9 expression, which is consistent with the observations in pancreatic cancer cells (25). Furthermore, EGF-1-induced expression of MMP-2 and MMP-9 was also markedly inhibited in ARK5 knockdown siARK5/SKOV3 cells. These results suggest that ARK5 functions upstream of MMP-2 and MMP-9, and directly regulates EGF-1-induced MMP expression.

The loss of epithelial markers and gain of mesenchymal markers are hallmarks of EMT. Numerous studies have indicated that EMT is a potential mechanism by which tumor cells acquire a more invasive and metastatic phenotype (26). Phippen et al (23) reported that elevated ARK5 is significantly associated with the mesenchymal subtype in high-grade serous ovarian cancer. The findings of the current study demonstrated that the expression of ARK5 is associated with EMT in SKOV3 cells. Knockdown of ARK5 repressed N-cadherin levels and increased E-cadherin expression. In addition, EGF-1 induced EMT features in Scr/SKOV3 cells, which was blocked by ARK5 knockdown. These data indicated that ARK5 knockdown promotes epithelial characteristics and reduces mesenchymal features. These findings are in accordance with the cellular migration and invasion properties of Scr/ARK5 and SiARK5/SKOV3 cells, which indicate that the hallmarks of EMT can be altered by ARK5.

This switch in cell epithelial and mesenchymal features is controlled by certain signaling pathways involved in cell invasion and metastasis (27). Recently, Zhang et al (22) demonstrated that ARK5 promoted EMT in ovarian cancer by inhibiting a microRNA-1181/homeobox A10 axis. In the current study, the function of Akt/mTOR signaling in regulating invasion of SKOV3 ovarian cancer cells was emphasized. As a member of the human AMPK family, ARK5 is directly activated by Akt dependent phosphorylation at serine residue on the regulatory domain (28). Akt/mTOR signaling is very important in promoting invasion and metastasis cancer cells via EMT and the expression of MMPs (29-31). ARK5 is a critical downstream effector of Akt and has critical roles in facilitating the invasion and metastasis of human malignant cancer cells (32). The findings of the current study demonstrated that ARK5 knockdown inhibited mTOR phosphorylation, regardless of EGF-1 stimulation. Considering that mTOR is directly phosphorylated by Akt (33), and the complexity and importance of the Akt/mTOR pathway, it is uncertain whether mTOR phosphorylation is directly modulated by ARK5 or indirectly by other factors that are regulated by ARK5. Collectively, the effects of ARK5 on SKOV3 cells invasion and metastasis may be attributed to its activation of the Akt/mTOR pathway (34). 
A

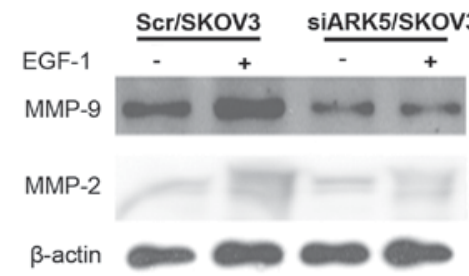

C

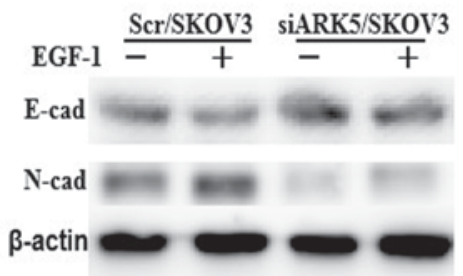

E

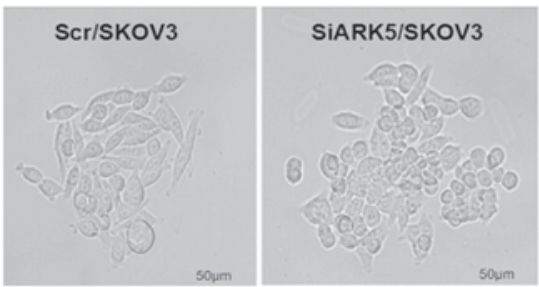

B
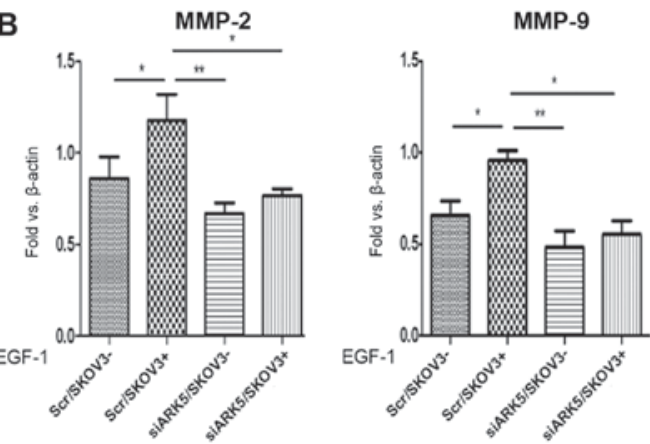

D

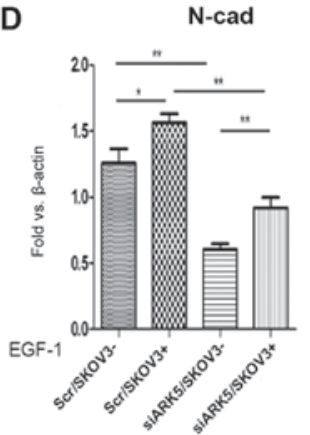

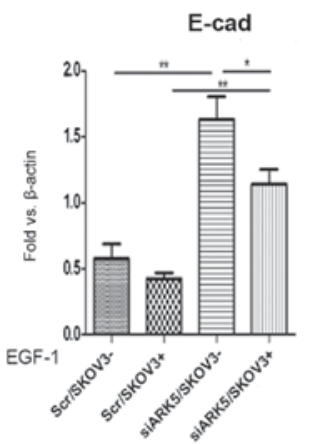

Figure 3. Reduction of ARK5 inhibited expression of MMP-2 and MMP-9, and reversed epithelia-mesenchymal transition. (A) Expressions of MMP-2 and MMP-9 in Scr/SKOV3 and siARK5/SKOV3 cells with or without EGF-1 (10 ng/ml) stimulation. (B) Statistical analysis of expressions of MMP-2 and MMP-9 in Scr/SKOV3 and siARK5/SKOV3 cells. (C) Expressions of N-cadherin and E-cadherin in Scr/SKOV3 and siARK5/SKOV3 cells with or without EGF-1 $(10 \mathrm{ng} / \mathrm{ml})$ stimulation. (D) Statistical analysis of expressions of N-cad and E-cad in Scr/SKOV3 and siARK5/SKOV3 cells. (E) Changes in cell morphology. ${ }^{*} \mathrm{P}<0.05,{ }^{* *} \mathrm{P}<0.01$. Scr, scramble; EGF-1, epidermal growth factor-1; siARK, ARK small interfering RNA; ARK5, AMPK-related protein kinase-5; MMP, matrix metallopeptidase; N-cad, N-cadherin; E-cad, E-cadherin.

A

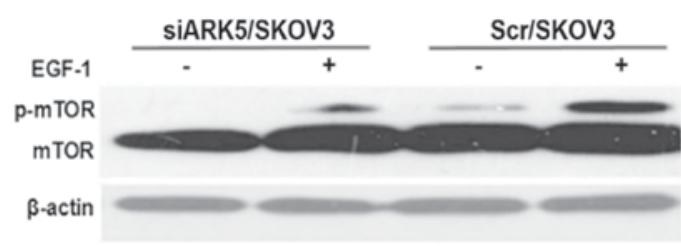

B

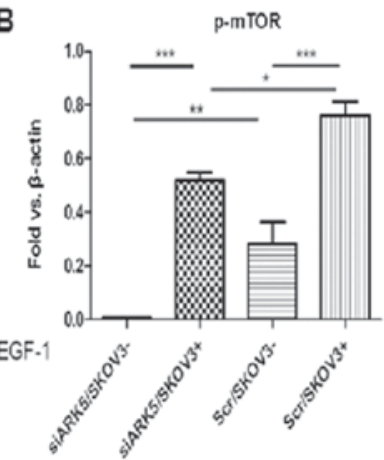

mTOR

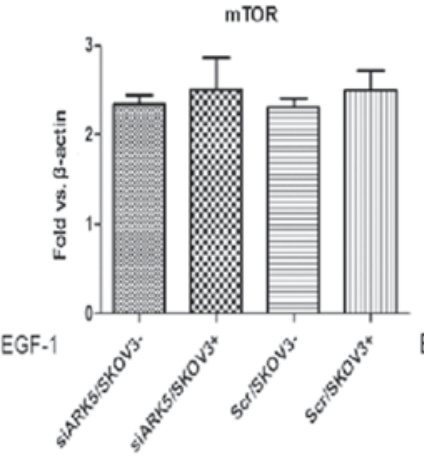

Figure 4. Reduction of ARK5 stabilizes Akt-mTOR signaling pathway. (A) Phosphorylation of mTOR was inhibited in siARK5/SKOV3 cells with and without EGF-1 (10 ng/ml) stimulation. (B) Statistical analysis of expressions of p-mTOR and mTOR in siARK5/SKOV3 cells with and without EGF-1 (10 ng/ml) stimulation. " $\mathrm{P}<0.05,{ }^{* *} \mathrm{P}<0.01,{ }^{* * *} \mathrm{P}<0.001$. siARK, ARK small interfering RNA; ARK5, AMPK-related protein kinase-5; Scr, scramble; EGF-1, epidermal growth factor-1; p-, phospho-; mTOR, mechanistic target of rapamycin kinase. 
In summary, human ovarian cancer exhibits high expression of ARK5 and knockdown of ARK5 significantly inhibits the invasive features of SKOV3 cells. In addition, the findings of the current study also indicate that ARK5 may mediate invasion of SKOV3 cells by increasing MMP-2 and MMP-9 expression and promoting EMT process via Akt/mTOR signaling. Thus, the findings have important implications for developing novel therapeutic targets for ovarian cancer.

\section{Acknowledgements}

Not applicable.

\section{Funding}

The present study was supported by Scientific Foundation of Shandong (grant no. ZR2015HL119), Scientific Foundation of Weifang Medical University (grant nos. 2017BSQD33 and 2017BSQD12), Scientific Foundation of Gansu Province (grant no. 2014GS02292), National Natural Scientific Foundation of China (grant nos. 81872163, 81672631, 81503108, 81072068 and 81001001), Shandong Province Study Abroad Program Foundation to LS and Weifang Medical University Study Abroad Program Foundation to XS and Domestic Visiting Scholar Foundation To RL. Scientific Foundation Of Shandong Administration Of Traditional Chinese Medicine (grant no. 2015-229). Scientific Foundation Of Shandong Education Administration (grant no. J17KA255).

\section{Availability of data and materials}

The datasets used and/or analyzed during the current study are available from the corresponding author on reasonable request.

\section{Authors' contributions}

SW, BZ and LS were involved in the conceptual design of the project and were major contributors in writing the manuscript. SW, SL and HW performed the western blot analysis, RT-PCR and Transwell assay, and were also major contributors in writing the manuscript. WL, YG, XW and CF performed substantive revision of the important content of the manuscript and made analysis and interpretation of data. CF and XS performed the chemotaxis index of cells under different concentrations of EGF-1. MC, RL and WS drafted the article and contributed to cell culture and cell transfection. All authors read and approved the final manuscript.

\section{Ethics approval and consent to participate}

The present study was conducted in accordance with the ethical standards in the Declaration of Helsinki (1975) and was approved by the Institutional Ethics Committee at Weifang Medical University All donors provided written informed consent.

\section{Patient consent for publication}

Not applicable.

\section{Competing interests}

The authors declare that they have no competing interests.

\section{References}

1. Bowtell DD: The genesis and evolution of high-grade serous ovarian cancer. Nat Rev Cancer 10: 803-808, 2010.

2. Siegel RL, Miller KD, Fedewa SA, Ahnen DJ, Meester RGS, Barzi A and Jemal A: Colorectal cancer statistics, 2017. CA Cancer J Clin 67: 177-193, 2017.

3. Ledermann JA, Embleton AC, Raja F, Perren TJ, Jayson GC Rustin GJS, Kaye SB, Hirte H, Eisenhauer E, Vaughan M, et al: Cediranib in patients with relapsed platinum-sensitive ovarian cancer (ICON6): A randomised, double-blind, placebo-controlled phase 3 trial. Lancet 387: 1066-1074, 2016.

4. Shevade A, Strogolova V, Orlova M, Yeo CT and Kuchin S: Mitochondrial voltage-dependent anion channel protein Por1 positively regulates the nuclear localization of saccharomyces cerevisiae AMP-activated protein kinase. mSphere 3: pii: e00482-17, 2018.

5. Zhang X, Lv H, Zhou Q, Elkholi R, Chipuk JE, Reddy MV, Reddy EP and Gallo JM: Preclinical pharmacological evaluation of a novel multiple kinase inhibitor, ON123300, in brain tumor models. Mol Cancer Ther 13: 1105-1116, 2014.

6. Cao D, Li D, Huang Y, Ma Y, Zhang B, Zhao C, Deng S, Luo M, Yin T, Wei YQ and Wang W: 5-azacytidine promotes invadopodia formation and tumor metastasis through the upregulation of PI3K in ovarian cancer cells. Oncotarget 8: 60173-60187, 2017.

7. Nehate C, Moothedathu Raynold AA and Koul V: ATRP fabricated and short chain polyethylenimine grafted redox sensitive polymeric nanoparticles for codelivery of anticancer drug and siRNA in cancer therapy. ACS Appl Mater Interfaces 9: 39672-39687, 2017.

8. Stubelius A, Andersson A, Islander U and Carlsten H: Ovarian hormones in innate inflammation. Immunobiology 222: 878-883, 2017.

9. Li L, Gong M, Zhao Y, Zhao X and Li Q: FOXK1 facilitates cell proliferation through regulating the expression of $\mathrm{p} 21$, and promotes metastasis in ovarian cancer. Oncotarget 8: 70441-70451, 2017.

10. Peng F, Zhong Y, Liu Y, Zhang Y, Xie Y, Lu Y, Zhang X and Li D: SPARC suppresses lymph node metastasis by regulating the expression of VEGFs in ovarian carcinoma. Int J Oncol 51: 1920-1928, 2017.

11. Mu QJ,Li HL, Yao Y,Liu SC, Yin CG and Ma XZ: Chromodomain helicase/ATPase DNA-binding protein 1-like gene (CHD1L) expression and implications for invasion and metastasis of breast cancer. PLoS One 10: e0143030, 2015.

12. Suzuki A, Lu J, Kusakai G, Kishimoto A, Ogura T and Esumi H. ARK5 is a tumor invasion-associated factor downstream of Akt signaling. Mol Cell Biol 24: 3526-3535, 2004.

13. Shi L, Sun X, Zhang J, Zhao C, Li H, Liu Z, Fang C, Wang X, Zhao C, Zhang X, et al: Gab2 expression in glioma and its implications for tumor invasion. Acta Oncol 52: 1739-1750, 2013.

14. Tang J, Qin Z, Han P, Wang W, Yang C, Xu Z, Li R, Liu B, Qin C, Wang $\mathrm{Z}$, et al: High Annexin A5 expression promotes tumor progression and poor prognosis in renal cell carcinoma. Int $\mathbf{J}$ Oncol 50: 1839-1847, 2017.

15. Wang Y and Zhou BP: Epithelial-mesenchymal transition in breast cancer progression and metastasis. Chin J Cancer 30: 603-611, 2011.

16. Xu T, Zhang J, Chen W, Pan S, Zhi X, Wen L, Zhou Y, Chen BW, Qiu J, Zhang Y, et al: ARK5 promotes doxorubicin resistance in hepatocellular carcinoma via epithelial-mesenchymal transition. Cancer Lett 377: 140-148, 2016.

17. Lue H, Thiele M, Franz J, Dahl E, Speckgens S, Leng L, Fingerle-Rowson G, Bucala R, Lüscher B and Bernhagen J: Macrophage migration inhibitory factor (MIF) promotes cell survival by activation of the Akt pathway and role for CSN5/JAB1 in the control of autocrine MIF activity. Oncogene 26: 5046-5059, 2007.

18. Lamouille S, Connolly E, Smyth JW, Akhurst RJ and Derynck R: TGF- $\beta$-induced activation of mTOR complex 2 drives epithelial-mesenchymal transition and cell invasion. J Cell Sci 125: 1259-1273, 2012 . 
19. Li M, Zheng C, Xu H, He W, Ruan Y, Ma J, Zheng J, Ye C and Li W: Inhibition of AMPK-related kinase 5 (ARK5) enhances cisplatin cytotoxicity in non-small cell lung cancer cells through regulation of epithelial-mesenchymal transition. Am J Transl Res 9: 1708-1719, 2017.

20. Kusakai G, Suzuki A, Ogura T, Kaminishi M and Esumi H: Strong association of ARK5 with tumor invasion and metastasis. J Exp Clin Cancer Res 23: 263-268, 2004.

21. Lu S, Niu N, Guo H, Tang J, Guo W, Liu Z, Shi L, Sun T, Zhou F, $\mathrm{Li} \mathrm{H}$, et al: ARK5 promotes glioma cell invasion, and its elevated expression is correlated with poor clinical outcome. Eur J Cancer 49: 752-763, 2013.

22. Zhang HY, Li JH, Li G and Wang SR: Activation of ARK5/miR-1181/HOXA10 axis promotes epithelial-mesenchymal transition in ovarian cancer. Oncol Rep 34: 1193-1202, 2015.

23. Phippen NT, Bateman NW, Wang G, Conrads KA, Ao W, Teng PN, Litzi TA, Oliver J, Maxwell GL, Hamilton CA, et al: NUAK1 (ARK5) is associated with poor prognosis in ovarian cancer. Front Oncol 6: 213, 2016.

24. Roh SA, Choi EY, Cho DH, Jang SJ, Kim SY, Kim YS and Kim JC: Growth and invasion of sporadic colorectal adenocarcinomas in terms of genetic change. J Korean Med Sci 25: 353-360, 2010.

25. Long H, Xie R, Xiang T, Zhao Z, Lin S, Liang Z, Chen Z and Zhu B: Autocrine CCL5 signaling promotes invasion and migration of CD133+ ovarian cancer stem-like cells via NF- $\mathrm{BB}$-mediated MMP-9 upregulation. Stem Cells 30: 2309-2319, 2012.

26. Liao TT and Yang MH: Revisiting epithelial-mesenchymal transition in cancer metastasis: The connection between epithelial plasticity and stemness. Mol Oncol 11: 792-804, 2017.
27. Shi L, Zhang B, Sun X, Lu S, Liu Z, Liu Y, Li H, Wang L, Wang X and Zhao C: MiR-204 inhibits human NSCLC metastasis through suppression of NUAK1. Br J Cancer 111: 2316-2327, 2014.

28. Suzuki A, Kusakai G, Kishimoto A, Lu J, Ogura T, Lavin MF and Esumi $\mathrm{H}$ : Identification of a novel protein kinase mediating Akt survival signaling to the ATM protein. J Biol Chem 278: 48-53, 2003.

29. Yu J, Tao S, Hu P, Wang R, Fang C, Xu Y, Qi D, Wei Z, Zhang J and Tan Q: CCR7 promote lymph node metastasis via regulating VEGF-C/D-R3 pathway in lung adenocarcinoma. J Cancer 8: 2060-2068, 2017.

30. Martin TA and Jiang WG: Anti-cancer agents in medicinal chemistry (formerly current medicinal chemistry-anti-cancer agents). Anticancer Agents Med Chem 10: 1, 2010.

31. Cheng $\mathrm{K}$ and Hao M: Metformin inhibits TGF- $\beta 1$-induced epithelial-to-mesenchymal transition via PKM2 relative-mTOR/p70s6k signaling pathway in cervical carcinoma cells. Int J Mol Sci 17: pii: E2000, 2016.

32. Suzuki A, Kusakai G, Kishimoto A, Shimojo Y, Miyamoto S, Ogura T, Ochiai A and Esumi H: Regulation of caspase- 6 and FLIP by the AMPK family member ARK5. Oncogene 23: 7067-7075, 2004.

33. Brunet A, Bonni A, Zigmond MJ, Lin MZ, Juo P, Hu LS, Anderson MJ, Arden KC, Blenis J and Greenberg ME: Akt promotes cell survival by phosphorylating and inhibiting a Forkhead transcription factor. Cell 96: 857-868, 1999.

34. Chen D, Liu G, Xu N, You X, Zhou H, Zhao X and Liu Q: Knockdown of ARK5 expression suppresses invasion and metastasis of gastric cancer. Cell Physiol Biochem 42: 1025-1036, 2017. 